\title{
A Conservative Management of Spontaneously Ruptured Liver Hydatid Cyst
}

\author{
Miriam Obeid $^{\mathrm{a}}$, Subhi Mansour ${ }^{\mathrm{a}}$, Mira Damouny ${ }^{\mathrm{a}}$, Amir Farah ${ }^{\mathrm{a}}$, Kenan Halloun ${ }^{\mathrm{a}}$, \\ Rozan Marjiyeh ${ }^{\text {a }}$ Jawad Ghalia ${ }^{a}$, Safi Khuria, b, c
}

\begin{abstract}
Hydatid disease is a zoonotic disease caused mainly by the cestode (tapeworm) Echinococcus granulosus, also known as hydatidosis. It is endemic mainly in areas where dog/horse breeding and sheep farming are common, such as Australia, New Zealand and the Mediterranean region. A rare, yet serious, complication of hydatid cyst of the liver is rupture, which could be spontaneous due to increased intra-cystic pressure or following a traumatic injury of the abdomen. Rupture into the peritoneal cavity may result in an anaphylactic reaction, usually treated by means of emergency surgical intervention. Herein, we present a case of a 55-year-old male patient, known to have liver hydatid cystic disease 3 years ago, who presented to other hospital with acute abdominal pain, tachycardia and hypotension. A computed tomography (CT) scan of the abdomen revealed ruptured liver hydatid cyst into the peritoneal cavity, with free intra-abdominal fluid and dissemination into the mesentery. The patient was treated successfully by conservative means including nil per os (NPO), intravenous fluids, noradrenaline and anthelminthic treatment by albendazole. Two months later the patient underwent laparoscopic resection of the mesenteric as well as the hepatic cyst. The presentation, diagnosis, course of treatment and follow-up are discussed in this report. Reviewing the current English literature reveals that this is the first case to report a successful initial conservative management of spontaneous intraperitoneal rupture of liver hydatid cyst.
\end{abstract}

Keywords: Liver hydatid cyst; Spontaneous intraperitoneal rupture; Conservative management

\section{Introduction}

Liver hydatid cystic disease, known also as echinococcal dis-

Manuscript submitted February 19, 2021, accepted March 11, 2021

Published online April 21, 2021

${ }^{a}$ General Surgery Department, Rambam Health Care Campus, Haifa, Israel bHPB and Surgical Oncology Unit, Rambam Health Care Campus, Haifa, Israel

${ }^{\mathrm{c} C o r r e s p o n d i n g ~ A u t h o r: ~ S a f i ~ K h u r i, ~ H P B ~ a n d ~ S u r g i c a l ~ O n c l o l o g y ~ U n i t, ~ G e n-~}$ eral Surgery Department, Rambam Health Care Campus, Haa'leya Hashniya, Haifa 31096, Israel. Email: s_khuri@rambam.health.gov.il

doi: https://doi.org/10.14740/gr1373 ease or hydatidosis, is a zoonotic disease caused mainly by the larvae of the parasitic tapeworm Echinococcus granulosus, and less by Echinococcus multilocularis [1]. It is prevalent among livestock who are the intermediate hosts and canids which are the definitive hosts. Hydatidosis is an endemic disease in areas where animal husbandry is common, such as the Mediterranean region (mainly Morocco, Spain, Turkey, Greece) Australia, New Zealand and South America [1]. The life cycle begins once the eggs are ingested and hatched in the host's intestines, in which they penetrate the intestinal wall by means of their oncospheres and consequently migrate via the portal or lymphatic systems invading various organs. Humans act as aberrant intermediate hosts if they ingest the eggs of the cestode by means of contaminated food or via fecal-oral route from an infected canid [2,3]. The liver (50$77 \%$ ), mainly the right hepatic lobe, followed by the lungs $(15-47 \%)$ is the most affected organs in humans. Other less common involved organs include the spleen, peritoneum and the brain [4]. Once in the organs, the oncospheres form hydatid cysts which are then filled with protoscolices and daughter cysts. In the case of cystic rupture, the protoscolices are evacuated and disseminated to other parts of the body to form secondary cysts [5]. In some cases, anaphylactic shock may also occur (12.5\%).

Liver echinococcosis is usually asymptomatic, presenting as an incidental clinical or radiological finding. Symptoms, mostly abdominal pain and discomfort, develop due to an increase in cyst size [6]. Complications of liver hydatid cyst disease are rare, yet life-threatening without early prompt management. Complications include rupture, spontaneous or traumatic, with or without dissemination and secondary bacterial infection [7]. The most common form of cyst rupture is into the biliary tree, followed by the peritoneal cavity, thoracic cavity through the diaphragm and into other intra-abdominal organs $[8,9]$.

Spontaneous intraperitoneal hydatid cyst rupture usually presents as an acute abdomen, managed by emergency surgery. Herein, we present a case of spontaneous rupture of liver echinococcosis, treated successfully by conservative measures.

\section{Case Report}

A 55-year-old male patient, known to have a 3-cm hydatid cyst 


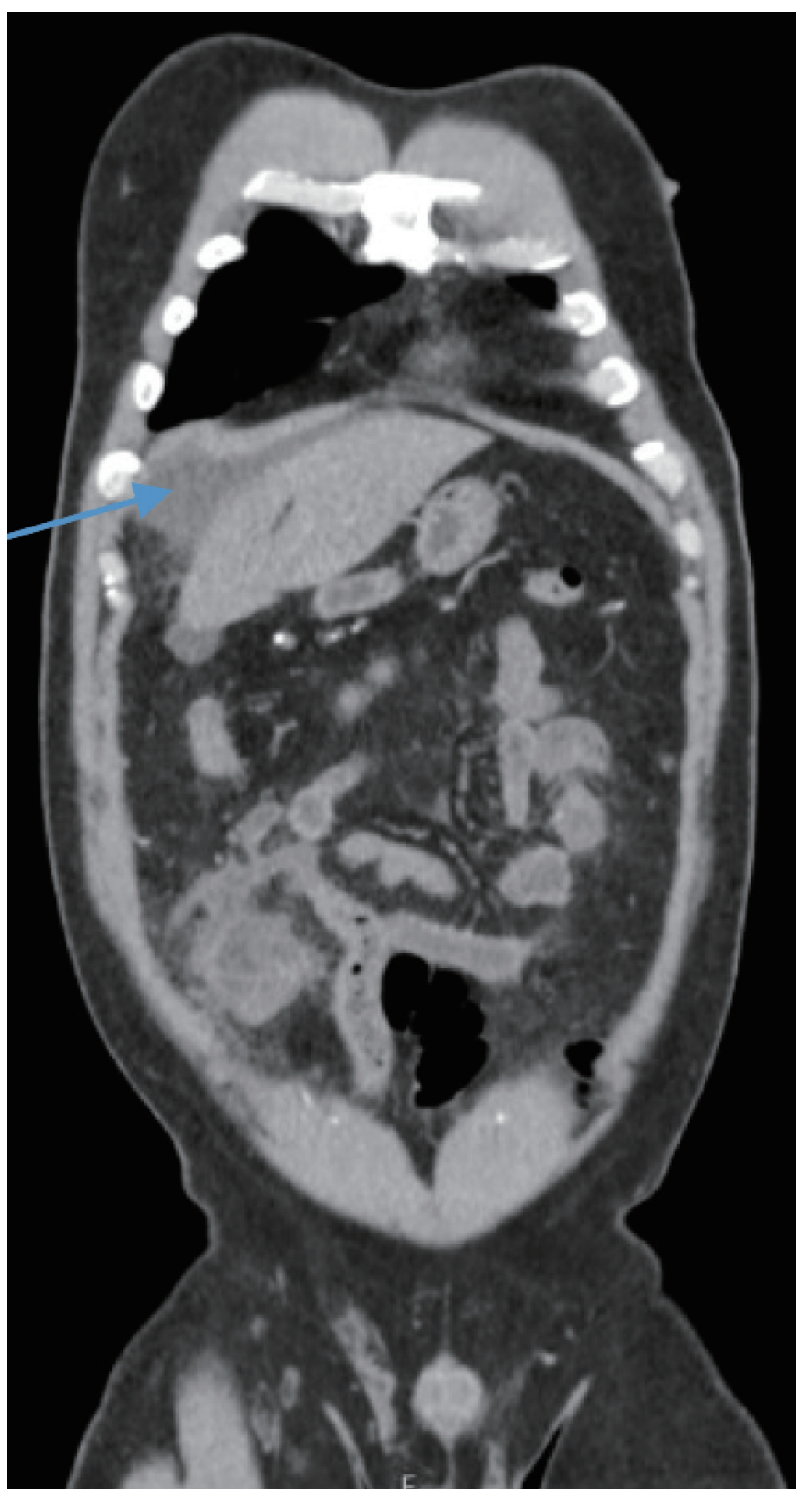

Figure 1. A coronal abdominopelvic CT scan shows hypodense cystic mass in segment 6 of the liver and an additional cystic mass in the mesentery of the small intestine with a moderate amount of free fluid in the abdomen (arrow). CT: computed tomography.

disease of the right lobe of the liver for 3 years was admitted to the emergency department of other peripheral hospital complaining of severe upper abdominal pain of 4-h duration. The pain had lasted for $4 \mathrm{~h}$, started abruptly, and was described as diffuse and sharp. He also suffered from nausea, recurrent vomiting, and general weakness. The patient denied any trauma to the chest or abdomen. In addition, the patient's background included a history of tobacco smoking, hypertriglyceridemia, and hypercholesterolemia.

On physical examination upon his admission, the patient's vital signs were abnormal, with tachycardia of 125 beats $/ \mathrm{min}$ and blood pressure of 100/60 mm Hg. An abdominal examination revealed upper abdominal diffuse tenderness with guarding. No abdominal mass was palpated. Digital rectal examina-

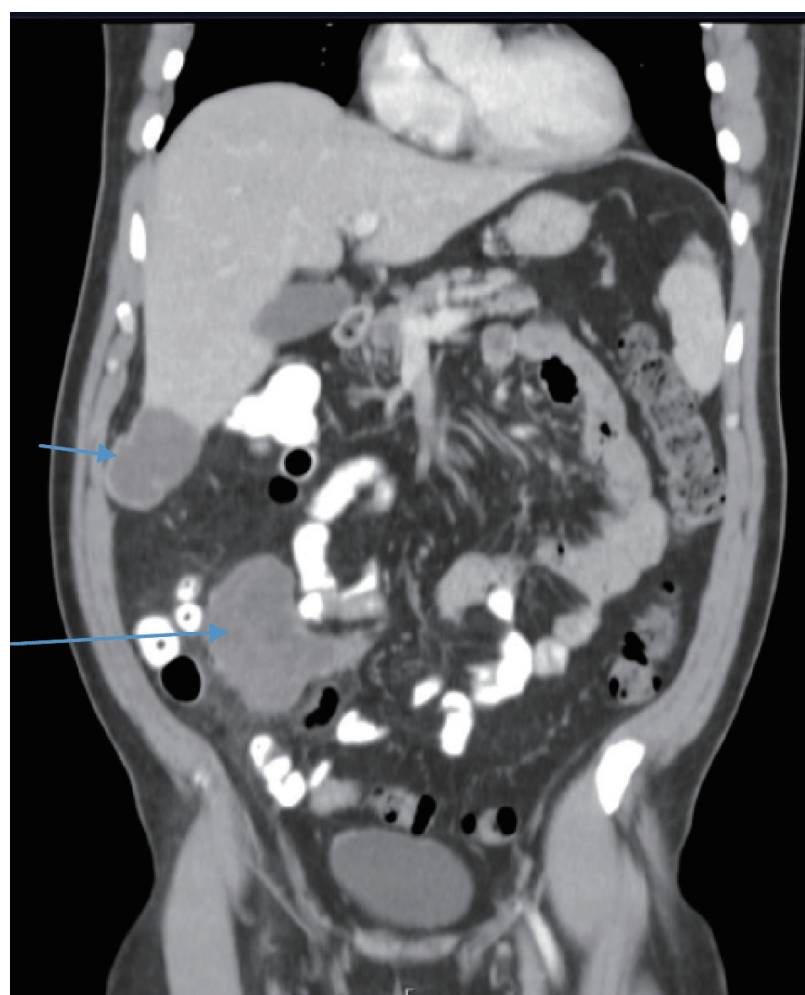

Figure 2. A coronal abdominopelvic CT scan 4 weeks later demonstrates the same findings of liver hydatid cyst (short arrow) and a wellorganized cystic mass at the small-bowel mesentery (long arrow), without free intraabdominal fluid. CT: computed tomography.

tion was normal. A complete blood count showed increased white blood cells of $18,000 \times 10^{9} / \mathrm{L}$, with $16 \%$ band neutrophils. Liver and kidney function tests were within normal limits. A computed tomography (CT) scan of the abdomen and pelvis revealed a $5-\mathrm{cm}$ hypodense cystic mass in segment 6 of the liver and an additional $10-\mathrm{cm}$ polycystic mass in the mesentery of the small intestine with moderate amount of free fluid in the abdomen and pelvis (Fig. 1). The patient was diagnosed with spontaneous intraperitoneal rupture of hepatic hydatid cyst with dissemination into the small-bowel mesentery, and he was transferred to our facility and admitted to the intensive care unit (ICU). Due to unavailability of operating room, conservative management by nil per os (NPO), rigorous intravenous fluids, noradrenaline and anthelminthic treatment by albendazole was initiated until an operating theater become available for surgical management. Few hours following his admission, the patient's hemodynamic status improved significantly, and he was weaned from noradrenaline support, thus, emergency surgery was cancelled. Four days later, the patient was discharged home in stable condition after ensuing resumption in his oral intake, with recommendation for continuation of oral albendazole ( $400 \mathrm{mg} / \mathrm{twice}$ daily) management for at least 6 weeks.

A follow-up abdominopelvic CT scan 4 weeks later showed the same findings of right hepatic lobe cyst of $5 \mathrm{~cm}$, multicystic mass of $10 \mathrm{~cm}$ at small-bowel mesentery, and a new small cystic mass of $3 \mathrm{~cm}$ at the right lower quadrant of 


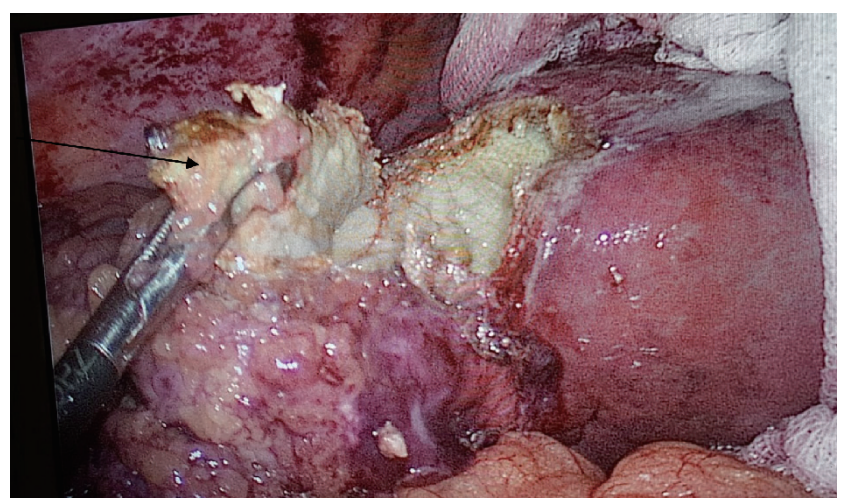

Figure 3. On laparoscopy, a partial pericystectomy of the hydatid cyst in the liver was performed (arrow), following careful cyst aspiration and injection of hypertonic (15\%) saline.

the abdomen (Fig. 2). Eight weeks later, the patient was admitted to our surgical department electively in stable condition for laparoscopic resection of the mesenteric cysts and pericystectomy of the hepatic cyst. He was asymptomatic and his physical exam was unremarkable. During the patient's hospitalization he underwent a laparoscopic surgery in which a $10-\mathrm{cm}$ echinococcal cyst which consisted of a daughter cyst was identified in the abdomen, wrapped in omentum and adhered to the small intestine along with small cyst of $3 \mathrm{~cm}$ in diameter at the right lower abdominal cavity. In addition, another echinococcal cyst adhered to the omentum was identified in segment 6 of the liver. A partial pericystectomy of the hydatid cyst in the liver with omentoplasty was performed, following careful cyst aspiration and injection of hypertonic (15\%) saline (Fig. 3), along with complete cystectomy of the lesions located in the small-intestine mesentery and right lower quadrant. His postoperative period was uneventful. The patient was discharged home on post-operative day 4 , and prescribed albendazole for 1 month. No recurrence or additional pathology was detected on CT of the abdomen and pelvis at the 3-, 6-, and 12-month follow-ups.

\section{Discussion}

Although most cases of liver hydatid disease remain silent over several years, fatal complications in the form of rupture or secondary infection may develop [6]. Our patient was known to have a hepatic hydatid cyst which was asymptomatic for years. Rupture may occur following trauma, including minor ones, or spontaneously $(1-8 \%)$ due to progressive increase in cyst size (larger than $10 \mathrm{~cm}$ in diameter). In our case the patient had no history of trauma [10]. Spontaneous cyst rupture usually occurs in younger age patients, with superficially located large lesion. The most common type of rupture is into the biliary tree, which accounts for $5-25 \%$ of cases, followed by intraperitoneal rupture in $3.2-16 \%[11,12]$. Intraperitoneal rupture of hepatic echinococcosis has a wide range of presentation, from mild abdominal pain to a life-threatening anaphylactic reaction, due to the dissemination of protoscoleces into the peritoneal cavity [13]. Our patient presented with hemodynamic instability along with acute abdomen.

Due to the aforementioned facts, prompt diagnosis of intraperitoneal rupture of hydatid cyst should be made. Diagnosis is usually made with radiological imaging using either abdominal ultrasound (US) or abdominopelvic CT scan, which has a sensitivity and specificity of up to $85 \%$ and $100 \%$, respectively [14]. CT scan findings suggestive of ruptured hydatid cyst usually include free intra-abdominal fluid, a detached membrane cyst and occasionally, dissemination into other organs. The previously mentioned CT scan findings were observed in our case.

Due to the risk of anaphylactic reaction, the gold standard therapy for intraperitoneal rupture of liver hydatid cyst is emergency surgery, which might be radical or conservative. In acute settings, conservative surgery in the form of unroofing is preferable, as it is much safer, easier and has a lower morbidity and mortality rates than the radical surgery (pericystectomy or hepatic resection), while still encompassing higher rates of future recurrence [15]. Conservative operations usually involve removal of all cyst contents with the cyst cavity, while washing the peritoneal cavity with scolicidal agents, mainly hypertonic saline $(3 \%, 15 \%$ or $30 \%)$.

Reviewing the current English literature reveals that all cases of spontaneous intraperitoneal ruptured hepatic echinococcosis were treated by emergency surgery. Herein, we report the first case to be treated successfully by initial conservative measures, followed by elective radical surgery 2 months later, with no clinical or radiological evidence of recurrence up to 1 year of follow-up. The rapid and significant hemodynamic improvement the patient presented, gave us the possibility to continue the conservative treatment.

\section{Conclusions}

The primary take-away message in this case report is although spontaneous intraperitoneal rupture of liver hydatid cyst might be life-threatening, an initial brief conservative management course in an ICU setting may be suggested in certain circumstances, in particular when hemodynamic improvement is promptly achieved. This might spare the patient suboptimal therapy (such as a conservative surgery), with higher future recurrence rates of hydatid cyst.

\section{Acknowledgments}

None to declare.

\section{Financial Disclosure}

There was no specific funding source to be mentioned.

\section{Conflict of Interest}

The authors have no conflict of interest to declare. 


\section{Informed Consent}

Written informed consent was obtained from the patient for publication of this case report and accompanying images.

\section{Author Contributions}

Miriam Obeid and Subhi Mansour contributed to the writing of the manuscript. Mira Damouny, Rozan Marjiyeh and Amir Farah contributed to the design. Kenan Halloun and Jawad Ghalia contributed to literature research and editing of the manuscript. Safi Khuri was the mentor, and contributed to critical revision of the manuscript.

\section{Data Availability}

The authors declare that data supporting the findings of this study are available within the article.

\section{References}

1. Nunnari G, Pinzone MR, Gruttadauria S, Celesia BM, Madeddu G, Malaguarnera G, Pavone P, et al. Hepatic echinococcosis: clinical and therapeutic aspects. World J Gastroenterol. 2012;18(13):1448-1458.

2. McIntyre N, Benhamou JP, Bircher J, Rizzetto M, Rodes J, Eds. Oxford Textbook of Clinical Hepatology. Vol. 1. 1991.

3. Sherlock S, Dooley J. Diseases of the liver and biliary system, 11th edition. 2002.

4. Beggs I. The radiology of hydatid disease. AJR Am J Roentgenol. 1985;145(3):639-648.

5. Kowalczyk M, Kurpiewski W, Zielinski E, Zadrozny D, Klepacki L, Juskiewicz W, Lasocki J, et al. A rare case of the simultaneous location of Echinococcus multilocularis in the liver and the head of the pancreas: case report analysis and review of literature. BMC Infect Dis. 2019;19(1):661

6. Alexiou K, Mitsos S, Fotopoulos A, Karanikas I, Tavernaraki K, Konstantinidis F, Antonopoulos P, et al. Complications of Hydatid Cysts of the Liver: Spiral Computed Tomography Findings. Gastroenterology Res. 2012;5(4):139-143.

7. Wen H, Aji T, Shao YM. Diagnosis and management against the complications of human cystic echinococcosis. Front Med China. 2010;4(4):394-398.

8. Marti-Bonmati L, Menor F, Ballesta A. Hydatid cyst of the liver: rupture into the biliary tree. AJR Am J Roentgenol. 1988;150(5):1051-1053.

9. Lewall DB, McCorkell SJ. Rupture of echinococcal cysts: diagnosis, classification, and clinical implications. AJR Am J Roentgenol. 1986;146(2):391-394.

10. Derici H, Tansug T, Reyhan E, Bozdag AD, Nazli O. Acute intraperitoneal rupture of hydatid cysts. World J Surg. 2006;30(10):1879-1883; discussion 1884-1875.

11. Gulalp B, Koseoglu Z, Toprak N, Satar S, Sebe A, Gokel Y, Sakman G, et al. Ruptured hydatid cyst following minimal trauma and few signs on presentation. Neth J Med. 2007;65(3):117-118.

12. Karavias DD, Vagianos CE, Kakkos SK, Panagopoulos CM, Androulakis JA. Peritoneal echinococcosis. World J Surg. 1996;20(3):337-340.

13. Kok AN, Yurtman T, Aydin NE. Sudden death due to ruptured hydatid cyst of the liver. J Forensic Sci. 1993;38(4):978-980.

14. Akcan A, Akyildiz H, Artis T, Ozturk A, Deneme MA, Ok E, Sozuer E. Peritoneal perforation of liver hydatid cysts: clinical presentation, predisposing factors, and surgical outcome. World J Surg. 2007;31(6):1284-1291.

15. Lo Casto A, Salerno S, Grisanti M, Mastrandrea G. Hydatid cyst of the liver communicating with the left colon. Br J Radiol. 1997; 70(834):650-651. 